\title{
Studies of Nanocomposites of Carbon Nanotubes and a Negative Dielectric Anisotropy Liquid Crystal
}

\author{
Parvathalu Kalakonda and Germano S Iannacchione \\ Additional information is available at the end of the chapter
}

http://dx.doi.org/10.5772/intechopen.73391

\begin{abstract}
The complex specific heat is reported over a wide temperature range for a negative dielectric anisotropy alkoxyphenylbenzoate liquid crystal (9OO4) and carbon nanotube (CNT) composites as a function of carbon nanotube concentration. It has been observed that the combination of nanotubes (CNT) and liquid crystal (LC) provides a very useful way to align CNTs and also dramatically increases the order in the liquid crystal performance, which is useful in liquid display technology (LCD). The calorimetric scans were performed between 25 and $95^{\circ} \mathrm{C}$ temperatures, first allowed cooling and then heating for CNT concentration ranging from $\varphi_{\mathrm{w}}=0$ to $0.2 \mathrm{wt} \%$. All 9OO4/CNT composite mesophases have transition temperatures about $1 \mathrm{~K}$ higher and a crystallization temperature $4 \mathrm{~K}$ higher as compared to the pure 9004 liquid crystal. A strongly first-order specific heat feature is observed, which is $0.5 \mathrm{~K}$ higher than in the pure $90 \mathrm{OO}$. The transition enthalpy for the composite mesophases is observed $10 \%$ lower than the pure liquid crystal. We interpret that these results arising from the LC-CNT surface interaction lead to pinning orientational order uniformly along the CNT, without pinning the position of the 9004 molecule. These effects of incorporating CNTs with LC are likely due to elastic coupling between CNT and LC. These effects of incorporating CNTs into LCs are likely due to an "anisotropic orientational" coupling between CNT and LC, the change in the elastic properties of composites and thermal anisotropic properties of the CNTs.
\end{abstract}

Keywords: carbon nanotubes, liquid crystal, chirality

\section{Introduction}

Composites of nanoparticles with liquid crystal represent a unique physical system, where properties of the constituents fully mix and may lead to show new behavior. As of now, 
traditional composites are well understood, the superposition of component of the composites interfacial interactions play a very important role in holding the composite together. As the filler size shrink, the surface area begins to dominate, leading to unique behavior of the composites. Carbon nanotubes (CNT) and liquid crystals (LC) are good examples of such components. For large number of applications, the challenge lies in the alignment and ordering of CNTs to take advantages of their highly anisotropic thermal characteristics. Carbon nanotubes have emerged as a new category of nanosized particles for incorporation into different liquid crystal systems, attracting favorable interest from both basic level science research and industrial applications [1]. As a result of the exceptional properties of carbon nanotubes, the novel materials can be envisioned that exhibit property enhancements at lower concentration than in conventional composite technology [2]. In the research area of polymer nanocomposites, reported property enhancements include enhanced mechanical performance [3, 4], higher thermal and electrical conductivity [5-7], and increased property of crystallization rate [8-12]. In addition to CNT's thermal, mechanical, and magnetic properties [13-22], their unique electrical properties make them potentially very useful materials for making nanoflexible electronic devices [2, 23-26]. Liquid crystals [27-29] are anisotropic fluids that exhibit thermodynamically stable phases between an isotropic liquid and a full, three-dimensionally ordered solid. In the nematic (N) phase, LCs show orientational order, and in the smectic-A $(\mathrm{SmA})$ phase, the rod-like molecules are arranged in layers with their long axes on average, normal to the layer planes. They show both orientational and partial translational order characterized by a quasi-1-d density wave. The smectic phases incorporate the structures of materials with diverse symmetry groups [30], such as that of smectic-C $(\mathrm{SmC})$, which has the layer tilting away from the director. In smectic- $\mathrm{B}(\mathrm{SmB})$ phase, the molecules show short-range hexagonal ordering within the layers but not from layer to layer, and it shows a short or longrange translational order. Higher order, lower symmetry, liquid crystalline materials have been studied because three of their industrial applications as well as important physical models of self-assembly $[29,30]$. When CNTs are dispersed in a liquid crystal, they can modify the physical properties and hence the phase behavior of the nanocomposite. Due to the specific surface anchoring between nanoparticle and LC, the nanoparticles can act either as nucleation sites for a given type of order or as disordering sites that stabilize the isotropic phase [31, 32]. However, if the local ordering effects of CNT surfaces are randomly arranged, this can lead to a random-field effect [33] and an overall disordering of the composites. Investigations have been made on liquid crystal nanocomposites using optical microscopy and differential scanning calorimetry (DSC), finding an enhancement of the isotropic to nematic phase transition temperature and revealing a "chimney type" phase diagram over a narrow range of CNT weight percent between 0.001 and $0.002 \mathrm{wt} \%$ [34]. In fact, researchers found that the addition of CNT into LC increases the clearing point of LCs because of the strong attraction of CNT and LC [35]. However, other studies have found disordering effects of CNT on LC phase transitions [31]. Other work has shown improved electro-optical switching properties of nanocomposites in thermotropic or lyotropic liquid crystals and CNTs [36-41]. In liquid crystals, the effect of carbon nanotubes on the phase ordering of LC/CNT composites depends on the surface coupling of the molecule and graphene surface, as well as the distribution of those surfaces. In fact, it is also observed that aligned CNTs can cause an increase of the orientational order in the LC $[42,43]$. Such composites have been proposed as memory devices 
by exploiting their nanoelectromechanical properties [44, 45]. Recently, investigations on LC/ CNT composites have shown that the CNTs can induce chirality in the bulk LC [46-48]. Most of the recent studies were focused on the nematic ordering and the isotropic $(\mathrm{I})$ to nematic $(\mathrm{N})$ phase transition behavior in LC/CNT composite scaffolds. In this study, we focus the phase transition behavior of the liquid crystal alkoxyphenylbenzoate (9OO4) doped with multiwall carbon nanotubes as a function of CNT weight percent. The incorporation of nanotubes in pure 9004 reveals that all mesophase transition temperatures and the crystallization transition temperatures shift upward. These results suggest that the interactions between molecular structure, dipole moment of liquid crystal, and graphene-like surface can allow the random dispersion of CNTs to promote both orientational and positional order. We interpret this effect in terms of pinning the director at the CNT surface with bulk-like scalar order parallel to the CNT long axis over distances that span multiple nematic domains while allowing the LC molecule to slide along the surface, accommodating four various positional orders. Our presentation is organized as follows: Following this introduction, Section 2 describes the preparation of the sample and modulated differential scanning calorimetry procedure, as well as the electroclinic procedure. Section 3 describes the calorimetric and electroclinic results of all phase transitions in the 9OO4/CNT system, Section 4 provides a discussion, and Section 5 provides conclusions of our work and future directions.

\section{Methodology}

\subsection{Material and sample preparation}

The liquid crystal 9004 is a phenyl benzoate containing an oxoester linkage group with two alkoxy end groups (see Figure 1). The molecular mass for 9004 is $\mathrm{Mw}=424.66 \mathrm{~g} / \mathrm{mol}$ with an extended molecular structure approximately $4 \mathrm{~nm}$ long and $0.8 \mathrm{~nm}$ wide. For 9004 , modeling suggests that due to the carbon = oxygen double bond in the oxoester linking group, the effective dipole moment of 9004 is approximately perpendicular to the long axis and average plane of the phenyl rings. The multiwall CNTs were obtained from Nanostructured and Amorphous Materials, Inc., and have an average outer diameter of $8-15 \mathrm{~nm}$, an inner diameter 3-5 nm, a length of 500-2000 nm, and a distribution of different chiral structures [46]. To reduce aggregation, a small amount of CNTs were dispersed first in acetone and shaken using a mixer for $30 \mathrm{~min}$, followed by sonication for 3 hours. The 9004 was added to the acetone $+\mathrm{CNT}$ mixture to

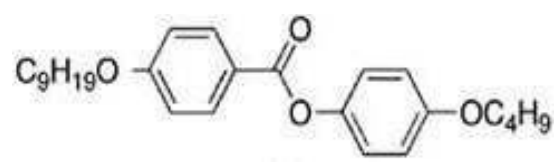

Figure 1. The member of the 4-n-alkoxy phenyl-40-m-alkoxy benzoate homolog series denoted nOOm. In this study, $\mathrm{n}=9$ and $\mathrm{m}=4$ (9OO4). Note that the negative dielectric anisotropy is due to the $\mathrm{O}-\mathrm{C}=\mathrm{O}$ group in the benzoate group linking the two phenyl rings. Molecular modeling suggests that the effective dipole moment of about 2.5 is pointing approximately perpendicular to the twisted phenyl rings, essentially out of the page [58]. 
achieve the desired final weight percent $\varphi_{\mathrm{w}}$ of CNTs. The mixture was then sonicated for 3 hours to facilitate good dispersion. Finally, the acetone was evaporated slowly and then allowed to degass under a modest vacuum in the isotropic phase of 9004 at $\sim 364 \mathrm{~K}$ (or $90^{\circ} \mathrm{C}$ ) for about 2 hours. Microbalance is used to mass the mixture of composites to ensure complete removal of acetone before sealed in the experimental sample cell. This process was continuously repeated to prepare 0 (pure 9OO4), 0.008, 0.010, 0.025, and $0.200 \mathrm{wt} \%$ CNT samples and tested uniform dispersion with the help of microscopy. On cooling, pure 9004 exhibits the phase sequence I - 87 - N - 73 - SmA - 62 - SmC - 50 - SmB - 35 - K (crystal), where temperatures are in Celsius; while on heating, melting occurs nearly $25 \mathrm{~K}$ higher, which is followed by a specific heat peak almost $2 \mathrm{~K}$ higher than the melting peak before entering the SmA phase. Further heating yields the $\mathrm{N}$ and I phases. Based on the imaginary specific heat $\Delta C^{\prime \prime} p$ behavior, the I-N transition is weakly first order, the N-SmA is continuous, the SmA-SmC is continuous, the SmC-SmB is first order, and the $\mathrm{SmB}-\mathrm{K}$ is strongly first order. The initial melting from crystal is strongly first order followed by a second, weakly first order, $\mathrm{Cp}$ feature that indicates the presence of a narrow temperature range having a smectic-like texture, as seen under polarizing microscopy (labeled SmX) between the $\mathrm{K}$ and SmA phase on heating [49]. Unfortunately, the polarizing microscope images could not distinguish which type of smectic is the SmX region.

\subsection{Modulated differential scanning calorimetry (MDSC)}

Modulated differential scanning calorimetry (MTDSC/MDSC) allows for the simultaneous measurement of the evolution of both the heat flow and heat capacity. It is essentially the combination of traditional ac-calorimetry and DSC. This method allows for measuring the total heat flow of material, as well as its nonreversible and reversible heat capacities. A detailed description of the MDSC method can be found elsewhere [50-57]. MDSC experiments were performed using a Model Q200 from TA Instruments, USA. Prior to all measurements, temperature calibration was done with a sapphire disc, under the same experimental conditions used for all 90O4/CNT samples. The analysis method used to extract the complex specific heat is based on linear response theory $[50,58]$. In general, a temperature oscillation is described as $T(t)=T_{0}+T_{0}{ }_{0} t+A_{T}$ $\sin (\omega t)$, where $T_{0}$ is the initial temperature at time $t=0, T$ indicates the absolute temperature at time $t, T_{0}^{*}$ represents the baseline temperature scan rate, $A_{T}$ is the temperature amplitude, and $\omega(\omega=2 \pi f)$ is the angular frequency of the temperature modulation. The rate of temperature is also time dependent and is given by equation $\mathrm{T}^{*}(\mathrm{t})=\mathrm{dT} / \mathrm{dt}=\mathrm{T}_{0}^{*}+\mathrm{Aq} \cos (\omega \mathrm{t})$, where Aq represents the amplitude of temperature modulation $\mathrm{r}(\mathrm{Aq}=\omega \mathrm{AT})$. Since the applied temperature rate consists of two components, $\mathrm{T}_{0}$ the underlying rate and $\mathrm{Aqcos}(\omega \mathrm{t})$ the periodic rate. The measured heat flow $\mathrm{H}_{\mathrm{F}}$ can also be separated into two components in response to these temperature rates. The periodic component can be described by $\mathrm{HFq}=\mathrm{A}_{\mathrm{HF}} \cos (\omega \mathrm{t}-\phi)$, where $\mathrm{A}_{\mathrm{HF}}$ represents the amplitude of the heat flow and $\phi$ is the phase angle between heat flow and temperature rate. The absolute value of the complex specific heat is written as $\mathrm{C} * \mathrm{p}=\mathrm{A}_{\mathrm{HF}} / \mathrm{mAq}$, where ' $\mathrm{m}$ ' is the mass of the sample. The phase angle $\phi$ requires a small calibration to account for finite six thermal conductivities of the sample and cell, see Ref. [57]. The real part of specific C'p and imaginary part of specific heat $C^{\prime \prime}{ }_{p}$ are then given by $C^{\prime} p=C * p \cos (\phi)$ and $C^{\prime \prime} p=C * p \sin (\phi)$, which allows for a consistent definition for the complex specific heat. Typically, under equilibrium conditions, $\mathrm{C}^{\prime \prime}$ $p=0$ after $\phi$ calibration. The appearance of a peak-like nonzero $C^{\prime \prime}{ }_{p}$ feature commensurate with 
a peak in the real part of the specific heat indicates that this feature is a first-order transition and involves a latent heat. The excess specific heats were determined in order to isolate the contribution from the various transitions. A linear baseline was used over the entire temperature scan range in order to determine $\Delta \mathrm{Cp}=\mathrm{C}_{\mathrm{p}}-\mathrm{C}_{\text {baseline }}$ for both the real and imaginary parts of specific heat components, though $C^{\prime \prime} p$ always exhibited a very shallow linear baseline that was very close to zero, indicating near equilibrium conditions for the experimental parameters used in this work. For specific heat features that are close in temperature, the wing of one peak is subtracted from the lower specific heat peak in order to isolate the excess specific heat of the lower temperature transition, denoted as $\delta \mathrm{Cp}=\Delta \mathrm{Cp}-\mathrm{C}_{\text {wing' }}$, where $\mathrm{C}_{\text {wing }}$ is a mimic function (polynomial) of the underlying wing. This calculation was only applied to the real component of the specific heat. The particular transition enthalpy component is simply the integration of the excess specific heat component over a consistent temperature range. Finally, for first-order transitions, the transition temperature $\left(\mathrm{T}_{\mathrm{IN}^{\prime}} \mathrm{T}_{\mathrm{CB}^{\prime}} \mathrm{T}_{\mathrm{BK}^{\prime}}, \mathrm{T}_{\mathrm{KX}}\right.$, and $\left.\mathrm{T}_{\mathrm{XA}}\right)$ is determined as the highest temperature of the two-phase coexistence region indicated by the onset of nonzero values of $\Delta C^{\prime \prime} p$. For continuous transitions, the transition temperature is taken as the $\Delta C^{\prime} p$ peak temperature. We have tested quasi-equilibrium parameter such as scan rate, temperature amplitude, and modulation time period by varying different values above and below specified values of nearly equilibrium.

\subsection{Electroclinic measurements}

Details of the electroclinic effect experimental set up are described elsewhere [59]. Briefly, a cell of thickness $\mathrm{d}=7.7 \pm 0.1 \mu \mathrm{m}$ was constructed from a pair of indium-tin-oxide-coated glass slides covered with a rubbed polyimide alignment layer. The cell was filled with $0.05 \mathrm{wt} \%$ CNT in 9OO4, which aligned in the planar direction. Light polarized at $22.5^{\circ}$ relative to the director orientation was incident on the cell, subsequently passing through the cell, an analyzer, and into a detector. On applying an ac-electric field $\mathrm{E}$ at frequency $\mathrm{f}=25 \mathrm{~Hz}$ across the cell, the director rotated in the cell's plane by an angle $\theta[\propto \mathrm{E}]$, resulting in an ac intensity component Iac at frequency f, as measured by a lock-in amplifier. It can be shown [59] that the ratio Iac/4Idc corresponds to the spatially averaged rms director rotation $\theta$, where Idc is the dc intensity measured at the detector. At each temperature, the electric field was ramped from 0 to $2.6 \times 106 \mathrm{~V} / \mathrm{m}$ (rms) over a time $450 \mathrm{~s}$, and the results were fitted to a straight line. The electroclinic coefficient is defined as ec $\equiv \mathrm{d} \theta / \mathrm{dE}$.

\section{Results}

For the pure 9004 , calorimetry shows that the I-N phase transition occurs at $\mathrm{T}_{\mathrm{IN}}=86.23^{\circ} \mathrm{C}$, the N-SmA phase transition at $\mathrm{T}_{\mathrm{NA}}=71.49^{\circ} \mathrm{C}$, the $\mathrm{SmA}-\mathrm{SmC}$ phase transition at $\mathrm{T}_{\mathrm{AC}}=61.5^{\circ} \mathrm{C}$, the SmC-SmB phase transition at $\mathrm{T}_{\mathrm{CB}}=49.38^{\circ} \mathrm{C}$, and $\mathrm{SmB}$-Cry phase transition at $\mathrm{TBK}=35.3^{\circ} \mathrm{C}$ in good agreement with literature values [49]. All the phase transitions are characterized by a distinct $C_{p}$ peaks at a nearly equilibrium scan rate of $\pm 0.3 \mathrm{~K} / \mathrm{min}$ (Figures 2-6). The strongly first-order melting followed by a second first-order $C_{p}$ feature indicates the presence of an intermediate phase (labeled SmX) between $\mathrm{K}$ and SmA on heating. The transition temperatures and enthalpies of pure 9004 on cooling and heating are presented in Tables 1 and 2. 


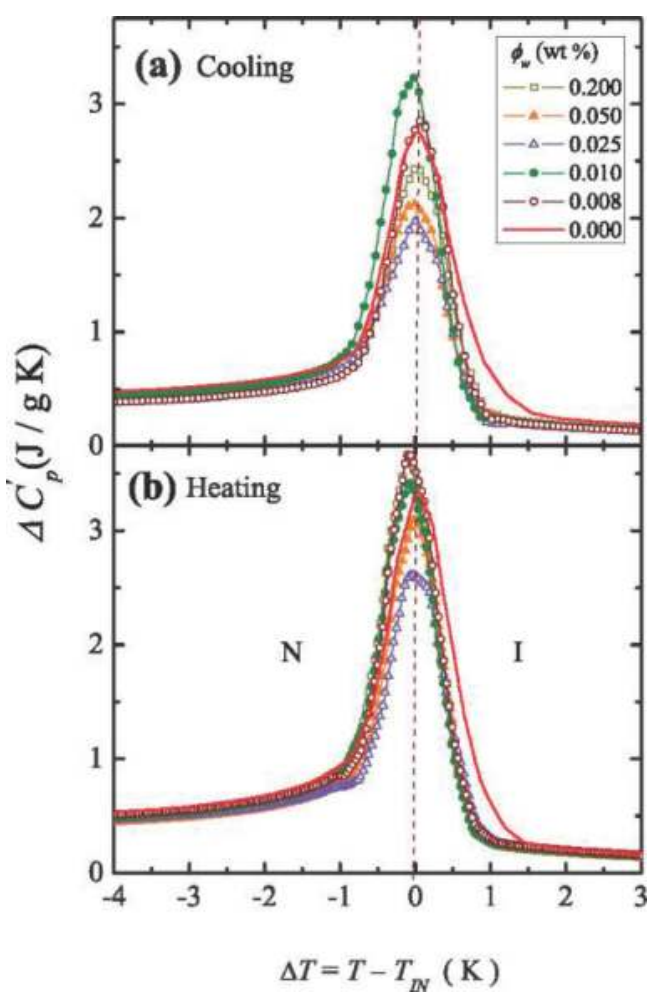

Figure 2. (a) Excess real part specific heat associated with the I-N phase transition as function of temperature about $T_{\text {IN }}$ on cooling. The definitions of the symbols are indicated in the inset. (b) Excess real part of specific heat associated with the I-N phase transition as function of temperature about $\mathrm{T}_{\mathrm{IN}}$ on heating.

For the I-N phase transition, the excess real specific heat signatures as a function of temperature about $\mathrm{T}_{\mathrm{IN}}$ for pure 9004 and 9OO4/CNT composite samples are shown in Figure 2. The $\Delta \mathrm{C}^{\prime} \mathrm{p}$ of $90 \mathrm{O} 4 / \mathrm{CNT}$ for the I-N transition phase is steeper and narrower than in the pure 9004, with the peak maximum nearer the high-temperature side of the coexistence region. The $\Delta C^{\prime} p$ and $C^{\prime \prime} p$ behavior are consistent on heating and cooling, as well as being reproducible after multiple thermal cycles. The $\Delta \mathrm{C}^{\prime} \mathrm{p}$ wings above and below the I-N transition match each other and that in pure 9004 on heating and cooling. The $\Delta C^{\prime} p$ peak height for cooling and heating scans within the two-phase $\mathrm{I}+\mathrm{N}$ coexistence region is about the same as that in pure 9004 up to $\varphi_{\mathrm{w}}=0.010$, decreasing markedly for the 0.025 sample and then rising with increasing $\varphi_{\mathrm{w}}$ up to the highest CNT content sample of $0.20 \mathrm{wt} \%$ studied.

For the N-SmA phase transition, the specific heat $\delta C^{\prime} p$ on cooling and heating as a function of temperature about $\mathrm{T}_{\mathrm{NA}}$ is shown in Figure 3 for bulk $9 \mathrm{OO} 4$ and $90 \mathrm{O} 4 / \mathrm{CNT}$ composite samples. For all samples, the N-SmA phase transition does not exhibit any special feature in the case of imaginary specific heat, indicating an apparent absence of latent heat, and indicates the continuous nature of the transition. The $\delta C^{\prime} p$ of the N-SmA transition for the composite $9004 / C N T$ 


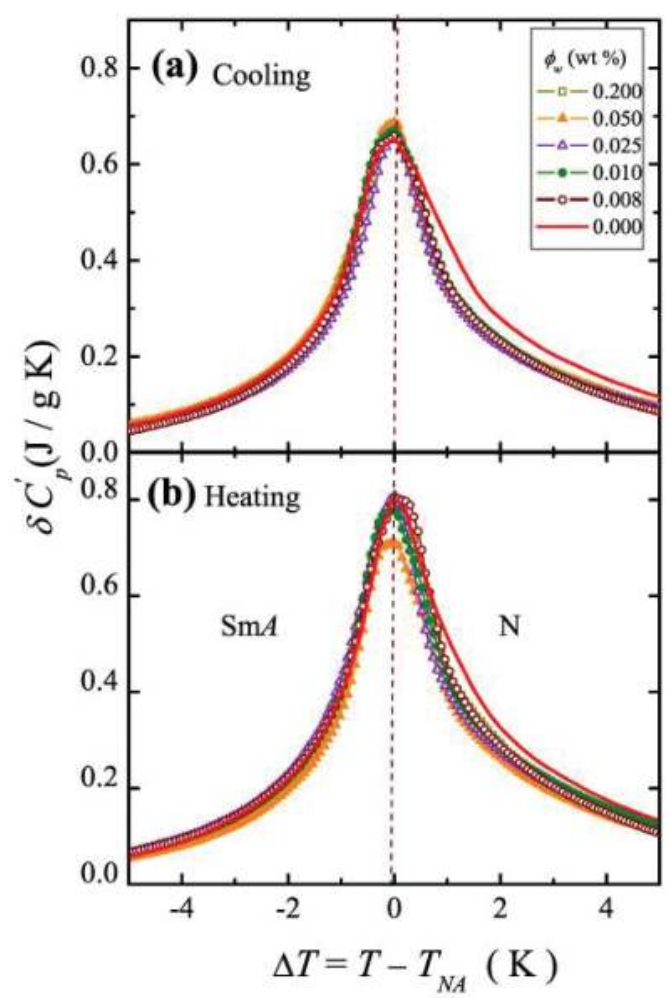

Figure 3. (a) The excess real part of specific heat associated with the N-SmA phase transition as function of temperature about $\mathrm{T}_{\mathrm{NA}}$ on cooling. (b) The excess real part specific heat associated with the N-SmA phase transition as function of temperature about $\mathrm{T}_{\mathrm{NA}}$ on heating. The definitions of the symbols are indicated in the inset.

samples overlay each other for all CNT concentrations $\varphi_{w}$ and for bulk 9004 on the SmA side while are systematically below bulk 9004 on the nematic side of the transition. The $\delta C^{\prime} p$ behavior does not show any features as a function of $\varphi_{w^{\prime}}$ and no power-law fits were attempted.

For the SmA-SmC phase transition, the excess specific $\delta C^{\prime} p$ as the function of temperature for pure 9004 and 9OO4/CNT composite samples is shown in Figure 4. The observed shape and continuous nature of the $\delta C^{\prime} p$ peak for pure 9004 are consistent with a Landau (mean-field) second-order transition [30] with no observed signature in $C^{\prime \prime} p$. The $\delta C^{\prime} p(A C)$ for the 90O4/ CNT samples, heights are the same as pure 9004 up to $0.025 \mathrm{wt} \%$ and then decrease for the 0.05 and $0.20 \mathrm{wt} \%$ samples. The step in $\delta C^{\prime} \mathrm{p}(\mathrm{AC})$ on the SmC-side for below $\mathrm{T}_{\mathrm{AC}}$ is $0.025 \mathrm{~J} / \mathrm{gK}$ for pure 9004 and all $9004 / C N T$ samples, independent of $\varphi w$.

The tilt susceptibility at the SmA-SmC transition was examined by measuring the electroclinic effect [59] in the LC/CNT mixtures. In the past, it has been shown that there is a nonzero enantiomeric excess for these CNTs, as well as a net chirality for CNTs from four other manufacturers [43]. When dissolved in a liquid crystal, the CNT imparts a net chiral environment to the 


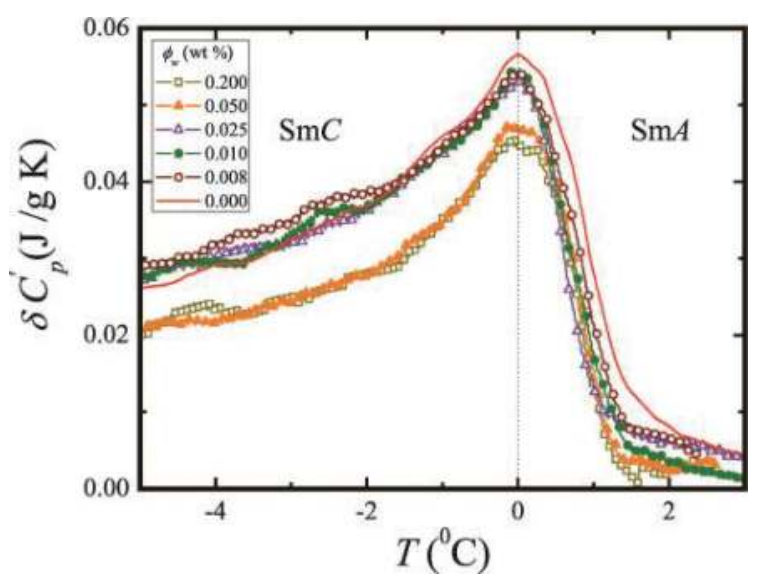

Figure 4. Excess real specific heat associated with the SmA-SmC phase transition as function of temperature about TAC on cooling for different CNT content sample. The definition of the symbols is given in the inset.

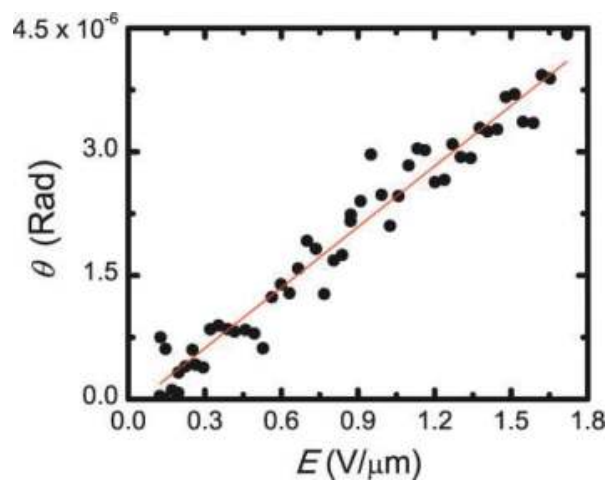

Figure 5. Electroclinic response is a function of electric applied field (E). Data collected at temperature $63.2^{\circ} \mathrm{C}$, approximately $1.2^{\circ} \mathrm{C}$ above SmA-SmC transition. Field was ramped over approximately $5 \mathrm{~min}$.

liquid crystal in the vicinity of the CNT surface. On application of an electric field, a surface electroclinic effect obtains, which requires the presence of a chiral symmetry environment and in which the director rotates by an angle $\theta \propto$ E. Because of the sufficiently high concentration of CNTs, the polarized light "sees" an average rotation of the liquid crystal director for the entire sample. Figure 5 shows the director rotation at temperature $63^{\circ} \mathrm{C}$ as the function applied filed.

The inverse electroclinic coefficient $\mathrm{e}_{\mathrm{c}}^{-1}$ is shown as a function of temperature for the $0.05 \mathrm{wt} \%$ 9OO4/CNT sample in Figure 6, approaching the SmC phase from the SmA. A three parameter (amplitude, $\mathrm{T}_{\mathrm{AC}}$ and susceptibility exponent) power-law fit of $\mathrm{e}_{\mathrm{c}}^{-1}$ versus temperature resulted in $\gamma=0.99 \pm 0.06$. Despite the localization of the chirality induced in the liquid crystal to within a few nanometers of the CNT surface [58], the susceptibility exponent clearly is mean-fieldlike and apparently unaffected by the presence of the nanotubes. This result suggests that the 


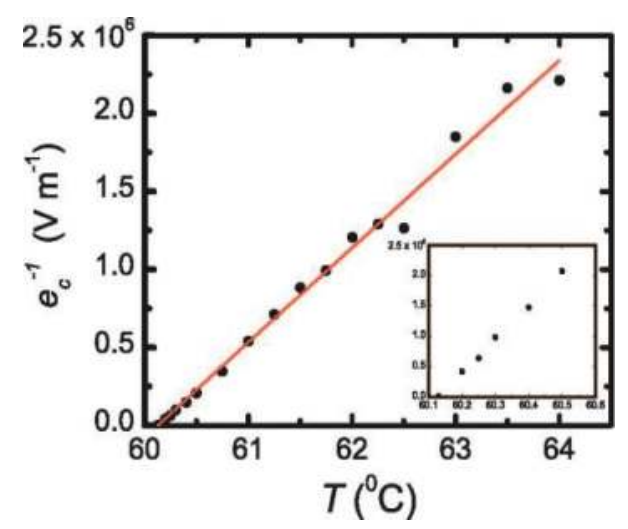

Figure 6. The inverse electroclinic coefficient $e-1 c$ is shown as a function of temperature for an applied electric field at frequency $25 \mathrm{~Hz}$ across the cell containing a $0.05 \mathrm{wt} \% 9004 / \mathrm{CNT}$ sample. The results were fitted a 3-parameter power law and resulted in a susceptibility exponent $\gamma=0.99 \pm 0.06$. Inset shows an expanded view of the inverse electroclinic coefficient for low temperature range.

\begin{tabular}{lllllllllll}
\hline & $\mathrm{T}_{\mathrm{IN}}(\mathrm{C})$ & $\mathrm{T}_{\mathrm{NA}}(\mathrm{C})$ & $\mathrm{T}_{\mathrm{AC}}(\mathrm{C})$ & $\mathrm{T}_{\mathrm{CB}}(\mathrm{C})$ & $\mathrm{T}_{\mathrm{BK}}(\mathrm{C})$ & $\mathrm{T}_{\mathrm{KX}}(\mathrm{H})$ & $\mathrm{T}_{\mathrm{KA}}(\mathrm{H})$ & $\mathrm{T}_{\mathrm{NA}}(\mathrm{H})$ & $\mathrm{T}_{\mathrm{IN}}(\mathrm{H})$ \\
\hline 0.000 & 86.19 & 71.27 & 61.10 & 49.38 & 35.00 & 60.96 & 63.55 & 71.46 & 86.48 \\
0.008 & 87.13 & 72.68 & 62.49 & 50.33 & 39.16 & 61.36 & 64.24 & 72.72 & 87.27 \\
0.010 & 87.14 & 72.69 & 62.50 & 50.26 & 39.34 & 61.45 & 64.34 & 72.85 & 87.27 \\
0.025 & 87.37 & 72.52 & 62.53 & 50.25 & 39.66 & 61.49 & 64.06 & 72.45 & 87.10 \\
0.050 & 86.92 & 72.50 & 62.28 & 50.17 & 39.85 & 61.42 & 64.00 & 72.62 & 87.35 \\
0.200 & 86.88 & 72.48 & 62.27 & 50.13 & 39.56 & 61.37 & 63.98 & 72.51 & 87.27 \\
\hline
\end{tabular}

CNT weight percent $\varphi_{w^{\prime}}$ the $I-N$ transition temperature $T_{I N}$, the $N-S m A$ transition temperature $T_{N A^{\prime}}$ the $K$-SmX transition temperature $T_{K X^{\prime}}$ the $\operatorname{SmX} X \mathrm{Sm} A$ transition temperature $T_{X A^{\prime}}$ the $\operatorname{Sm} A$-SmC transition temperature $T_{A C^{\prime}}$ the $\operatorname{SmC}-\operatorname{Sm} B$ transition temperature $T_{C B^{\prime}}$ and the $S m B-K$ transition temperature $T_{B K}$ are shown. All temperatures are given in Celsius.

Table 1. Summary of the transition temperatures for pure 9004 and all 9OO4/CNT samples on cooling $(\mathrm{C})$ and heating $(\mathrm{H})$.

director rotation extends a length scale $\xi$, comparable to the SmC correlation length, into the bulk liquid crystal from the narrow chiral region immediately surrounding the CNTs, which is the region that responds directly to the applied field. As an aside, we note that although $\mathrm{T}_{\mathrm{AC}}$ was obtained for this concentration of nanotubes, it is not possible to compare it with the transition temperature in the absence of nanotubes, for which chirality would be absent, and no electroclinic effect would be present.

For the SmC-SmB-K phase transition sequence on cooling, the $\Delta \mathrm{C}^{\prime} \mathrm{p}$ and $\mathrm{C}^{\prime \prime} \mathrm{p}$ signatures for pure 9004 and $9004 / \mathrm{CNT}$ composite samples over a range from +4 to $-10 \mathrm{~K}$ about $\mathrm{T}_{\mathrm{CB}}$ are shown in Figure 7. All peak signatures remain sharp, while the peak height of the SmC-SmB phase transition decreases with increasing $\varphi_{\mathrm{w}}$. The SmC-SmB transition is marked by a strongly first-order specific heat signature at $\mathrm{T}_{\mathrm{CB}}=49.38^{\circ} \mathrm{C}$, with the imaginary part being much larger than the real part. The total transition enthalpy $\Delta \mathrm{H}_{\mathrm{CB}}$ decreases slightly, while $\Delta \mathrm{H}_{\mathrm{BK}}$ increases 


\begin{tabular}{lllllllllllll}
\hline$\varphi_{\mathrm{w}}$ & $\delta H_{\mathrm{IN}}^{*}(\mathrm{C})$ & $\left.\delta \mathrm{H}_{\mathrm{N}}^{\prime \prime} \mathrm{C}\right)$ & $\delta H_{\mathrm{NA}}(\mathrm{C})$ & $\Delta H_{\mathrm{CB}}(\mathrm{C})$ & $\Delta H_{\mathrm{BK}}(\mathrm{C})$ & $\Delta H_{\mathrm{T}}$ & $\Delta H_{\mathrm{KX}}(\mathrm{H})$ & $\Delta H_{\mathrm{KA}}(\mathrm{H})$ & $\Delta H_{\mathrm{NA}}(\mathrm{H})$ & $\left.\Delta H_{\mathrm{IN}} \mathrm{H}\right)$ & $\Delta H_{\mathrm{T}}$ \\
\hline 0.000 & 3.9 & 0.95 & 3.1 & 35.9 & 35.9 & 80.2 & 72.0 & 32.0 & 3.0 & 4.9 & 83.4 \\
0.008 & 3.8 & 0.99 & 2.6 & 38.9 & 38.9 & 84.1 & 69.2 & 17.1 & 2.9 & 5.1 & 85.7 \\
0.010 & 4.3 & 0.96 & 2.6 & 39.7 & 39.7 & 84.4 & 67.4 & 13.6 & 2.9 & 4.8 & 83.9 \\
0.025 & 3.2 & 0.69 & 2.6 & 29.1 & 29.1 & 70.1 & 67.8 & 10.5 & 2.9 & 4.2 & 75.0 \\
0.050 & 3.4 & 1.01 & 2.8 & 31.2 & 31.2 & 72.4 & 61.9 & 10.3 & 2.8 & 4.4 & 85.3 \\
0.200 & 3.6 & 1.03 & 2.7 & 27.4 & 27.4 & 70.0 & 62.0 & 12.7 & 2.9 & 5.0 & 74.6 \\
\hline
\end{tabular}

CNT weight percent $\varphi_{w w^{\prime}}$ the $I-N$ transition $\delta H^{*}{ }_{I N}$ real enthalpy, imaginary enthalpy $\delta H^{\prime \prime}{ }_{I N^{\prime}}$ the $N$-Sm $A$ transition enthalpy $\delta H_{N A^{\prime}}$ the $K-\operatorname{SmX}$ total transition enthalpy $\Delta H_{K X^{\prime}}$ the $\operatorname{SmX}-\operatorname{Sm} A$ total transition enthalpy $\triangle H X A$, the $\operatorname{SmC}-\operatorname{Sm} B$ total transition enthalpy $\Delta H_{C B^{\prime}}$ and the $\operatorname{Sm} B-K$ total transition enthalpy $\Delta H_{B K}$ are shown. All enthalpies are given in joules per gram. The cumulative sum of all total transition enthalpies $\Delta H_{T}$ on cooling $(\mathrm{C})$ and heating $(\mathrm{H})$ is also shown.

Table 2. Summary of the transition enthalpies for pure 9004 and all 9OO4/CNT samples on cooling and heating.

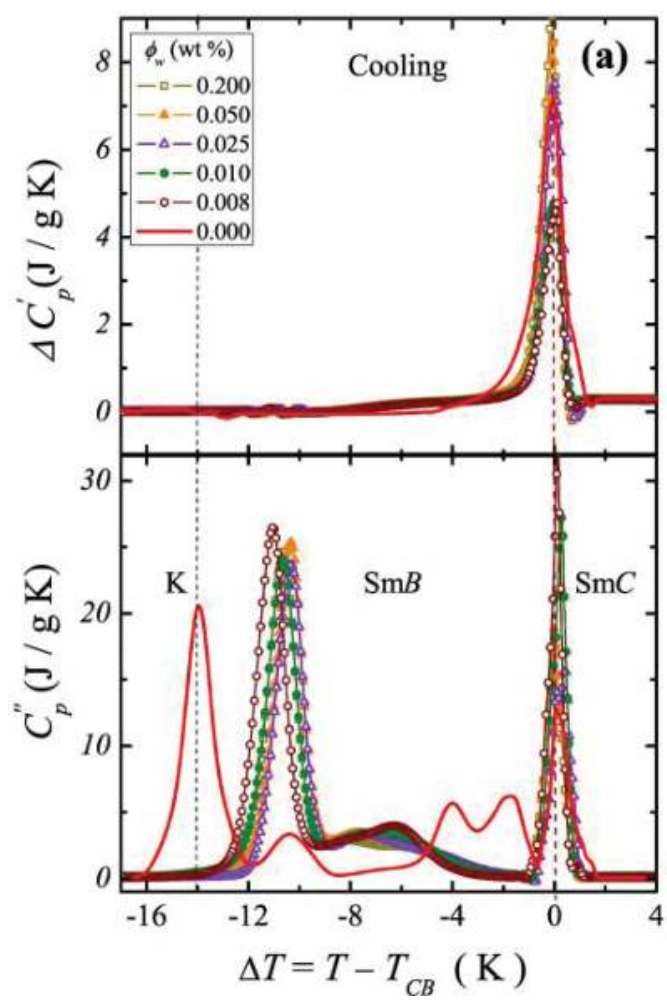

Figure 7. (a) Excess real specific heat associated with the SmC-SmB phase transition as function of temperature about $T_{C B}$ on cooling. The definition of the symbols is given in the inset. (b) Imaginary specific heat as function of temperature about $T_{C B}$ on cooling. 


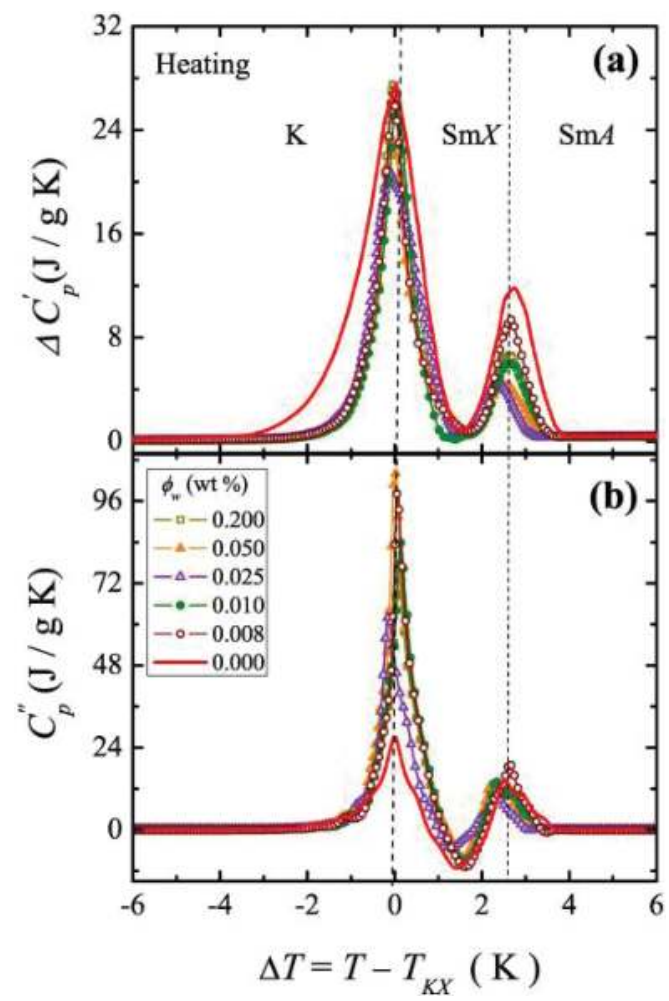

Figure 8. (a) Excess real-specific heat associated with the $\mathrm{K}-\mathrm{SmX}$-SmC phase transition as function of temperature about $T_{X A}$ on heating. The definition of the symbols is given in the inset. (b) Imaginary specific heat as function of temperature about $T_{X A}$ on heating.

slightly with increasing $\varphi_{\mathrm{w}}$. Similarly, the imaginary part is much larger than the nearly nonexistent $\Delta C^{\prime} p$ for the SmB-K transition. This is consistent with both the SmC-SmB and SmB-K phase transitions being strongly first order, as expected. Note that the $C^{\prime \prime} p$ behavior observed in the SmB phase are due to a very slow phase conversion to the SmB from the SmC.

For all samples, upon heating under continuous quasi-equilibrium conditions, the crystal phase superheats slightly until a strongly first-order specific heat feature is observed. Upon further heating, a second first-order feature is seen before the SmA phase appears. Figure 8 shows pure 9004 and $9004 / \mathrm{CNT}$ composite samples over a range from -6 to $+6 \mathrm{~K}$ about $\mathrm{T}_{\mathrm{XA}}$. The K-SmX $\Delta \mathrm{C}_{\mathrm{p}}$ peak is narrower for composite samples, except for the $\varphi_{\mathrm{w}}=0.025 \mathrm{wt} \%$ sample, as compared to the pure 9004 . The melting $\Delta C^{\prime} p$ peak generally decreases in amplitude with an increase in the $C^{\prime \prime} p$ peak. The total transition enthalpy of $\Delta \mathrm{H}_{\mathrm{KX}}=72 \mathrm{~J} / \mathrm{g}$ for pure 9004 decreases slightly for the 9OO4/CNT samples with increasing $\varphi_{\mathrm{w}}$. The $\Delta \mathrm{H}_{\mathrm{XA}}=32 \mathrm{~J} / \mathrm{g}$ and strongly decreases with $\varphi_{\mathrm{w}}$. Interestingly, the second feature specific heat peak height decreases with increasing $\varphi_{w}$ and suggests that this feature is not due to residual crystal melting. Because 
of the magnitude of the enthalpy involved, it is possible that this feature is a transition into an intermediate smectic phase before heating into the SmA phase. We denote this phase as SmX and are unclear from polarizing microscopy image as to whether this phase is SmB or SmClike, since the melting occurs very near $\mathrm{T}_{\mathrm{AC}}$ on cooling.

While the calorimetric and electroclinic behavior of the phases and phase transitions for the 9OO4/CNT samples are clearly retaining the character found in pure 9004, the phase boundaries have more shrinking changes due to the CNT. The transition temperature changes from pure 9004 for all 9OO4/CNT samples are shown in Figure 9. On cooling, the I-N transition temperature in the $9004 / \mathrm{CNT}$ samples shift upward by nearly a constant $+1.18 \mathrm{~K}$ compared to that in pure 9OO4, while the two-phase I $+\mathrm{N}$ coexistence width shrink with a constant

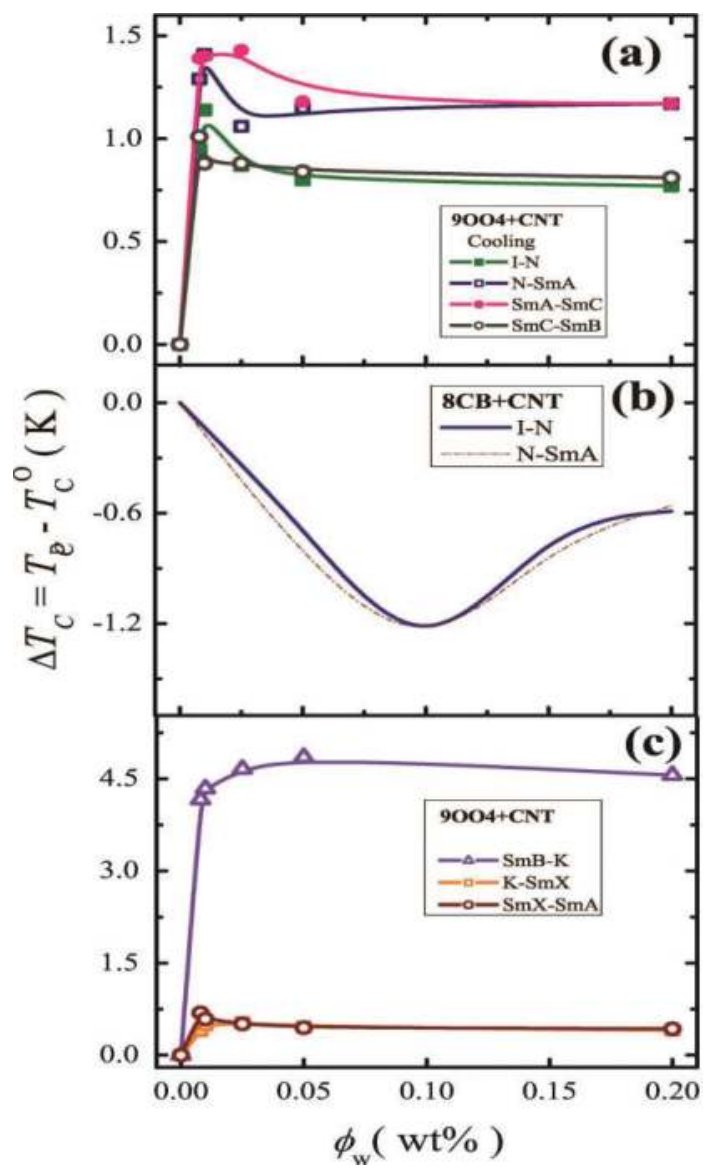

Figure 9. (a) The phase transition temperature shifts for the $I-N(\bullet), N-\operatorname{SmA}\left({ }^{\circ}\right), \operatorname{SmA}-\mathrm{SmC}(\bullet)$, and $\operatorname{SmC}-\operatorname{SmB}\left({ }^{\circ}\right)$ in $9004 / C N T$ samples and the $I-N(-)$. (b) N-SmA (-- ) transition temperature shift for $8 \mathrm{CB} / \mathrm{CNT}$ as a function of $\varphi w$. (c) The phase transition temperature shifts of the SmB-K $(\Delta), \mathrm{K}-\mathrm{SmX}\left({ }^{\circ}\right)$, and $\mathrm{SmX}-\mathrm{SmA}\left({ }^{\circ}\right)$ for the 9OO4/CNT samples as a function of $\varphi w$. 
$\sim 1.5 \mathrm{~K}$ in 9OO4/CNT over this range of $\varphi_{\mathrm{w}}$. For the N-SmA, transition temperature in 9OO4/ CNT shifts upward by $+1.31 \mathrm{~K}$ compared to pure 9004 , shrinking the nematic temperature range $\Delta \mathrm{T}_{\text {nem }}=\mathrm{T}_{\mathrm{IN}}-\mathrm{T}_{\mathrm{NA}}$ by $+0.3 \mathrm{~K}$ for all $\varphi_{\mathrm{w}}$. The SmA-SmC transition temperature in 9OO4/ CNT shifts upward by about $+1.4 \mathrm{~K}$, widening the $\mathrm{SmA}$ range by $+0.4 \mathrm{~K}$ for all $\varphi w$. The $\mathrm{SmC}-$ $\mathrm{SmB}$ transition temperature shifts upward by $+1.01 \mathrm{~K}$, widening the $\mathrm{SmC}$ range slightly for all $\varphi_{\mathrm{w}}$ studied. Finally on cooling, the SmB-K crystallization shifted upward by the largest, $+4 \mathrm{~K}$ for all $\varphi \mathrm{w}$, narrowing the $\mathrm{SmB}$ range by $3 \mathrm{~K}$. On heating, the $\mathrm{K}-\mathrm{SmX}$ melting superheats an additional $+0.4 \mathrm{~K}$, similarly for the SmX-SmA boundary compared to pure 9004 for all $\varphi w$ samples. The $\mathrm{T}_{\mathrm{NA}}$ on heating is $+1.2 \mathrm{~K}$, and the TIN is $+0.8 \mathrm{~K}$ higher than in pure 9004 , narrowing the nematic range $\Delta \mathrm{T}_{\text {nem }}$ by $0.4 \mathrm{~K}$, similar to that seen on cooling.

\section{Discussion}

It is useful to compare these results with another high-resolution phase transition study on 8CB/CNT nanocomposites, which also used near equilibrium calorimetric conditions and identical mixing method of CNTs from the same source [31]. The 8CB is a typical rod-like molecule, with biphenyl core, to which an aliphatic tail and a polar cyano head group are attached, whereas 9004 has a benzoate group, linking the two phenyl rings and having alkoxy end tails (see Figure 1). The transition temperatures and enthalpies of the isotropic to nematic and nematic to smectic-A phase transitions in 90O4/CNT and 8CB/CNT systems [31] are shown in Figures 9 and 10. In the 8CB/CNT system, both transition temperatures shift downward by $\sim 1.5 \mathrm{~K}$ with increasing $\varphi \mathrm{w}$, with a nonlinear $\varphi_{\mathrm{w}}$ dependence, while $\Delta$ Tnem remains constant as seen by the dashed and dash-dotted lines in panel (a) of Figure 9. Clearly, the same surface and distribution of CNTs for $8 \mathrm{CB}$ produce disordering effects on orientational order shifting both the I-N and N-SmA transitions. The transition enthalpies of the I-N and N-SmA phase transitions in the $8 \mathrm{CB} / \mathrm{CNT}$ system increase over a broad range of $\varphi_{\mathrm{w}}$ and then remain constant for higher $\varphi_{\mathrm{w}}$. The total transition enthalpy of $8 \mathrm{CB} / \mathrm{CNT}$ system has been interpreted as the sum of the pure transition contribution and a random field induced distortion energy. Apparently, given the near constant decrease of the enthalpy of the $9004 / \mathrm{CNT}$, the ordering is bulk like with a reduction perhaps due to suppression of long-wavelength director fluctuations. If the enthalpy suppression of the I-N and N-SmA in 9OO4/CNT was due to surface pinning, it should scale with CNT surface area, approximately linear in $\varphi_{\mathrm{w}}$ for these low concentrations, which is not observed. This suggests a different surface interaction for the 9004 than $8 \mathrm{CB}$ molecule with the CNT. The electroclinic effect result suggests that the director rotation $\theta$ extends a length scale $\xi$, comparable to the SmC correlation length, into the bulk liquid crystal forming a chiral region immediately surrounding the CNTs. This chiral region responds to the applied field, resulting in a tilt over the entire SmC correlation region. On cooling, the 9OO4/CNT SmB-K phase transition temperature increases $4 \mathrm{~K}$, and $\mathrm{K}-\mathrm{SmX}$ phase transition temperature increases $0.5 \mathrm{~K}$ on heating for all $\varphi \mathrm{w}$, while the total transition enthalpy remains constant on heating and cooling (see Figures 9 and 10). It is important to note that all experimental results presented here are reproducible over repeated scans. 


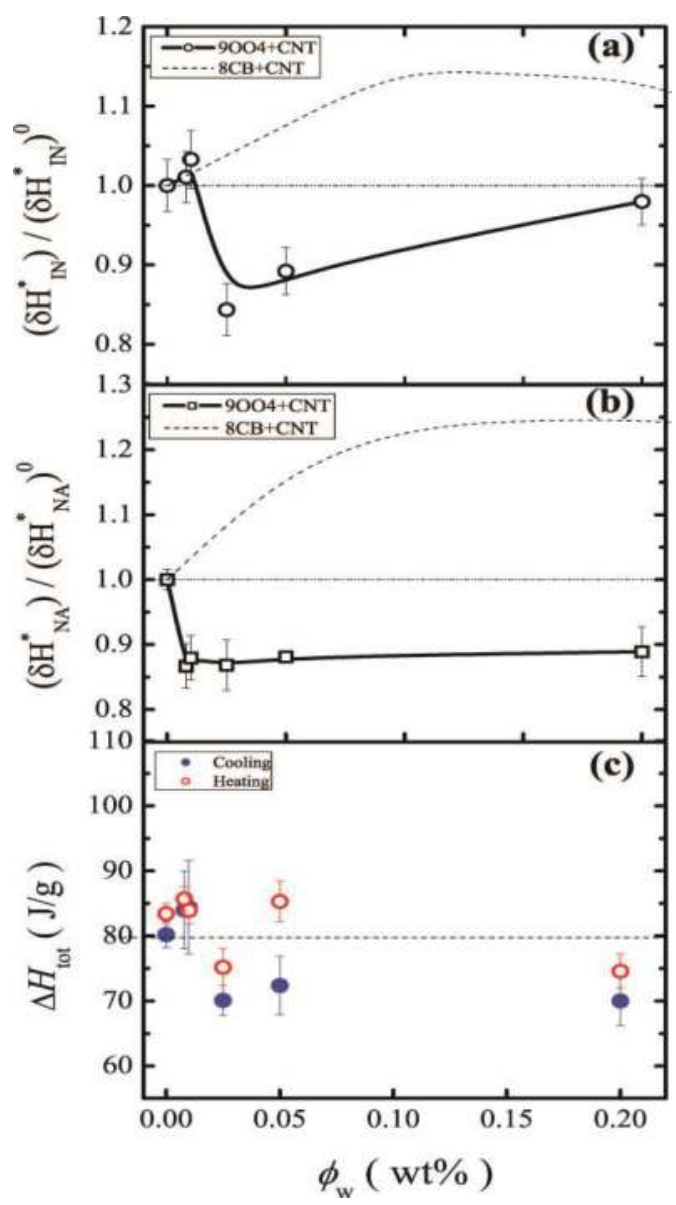

Figure 10. (a) The $I-N$ average fractional effective transition enthalpy for $9004 / \mathrm{CNT}\left(^{\circ}\right)$ and $8 \mathrm{CB} / \mathrm{CNT}(---)$ as a function of $\varphi w$. (b) The NSmA average fractional effective transition enthalpy for $9004\left({ }^{\circ}\right)$ and $8 \mathrm{CB}(---)$ nanocomposites as a function of $\varphi w$. (c) The effective total transition enthalpy on heating $\left({ }^{\circ}\right)$ and cooling $(\bullet)$ for the $9004 / \mathrm{CNT}$ samples as the function of $\varphi w$.

From the calorimetric observations presented here, the effect of CNTs on the phase transitions of 9004 apparently enhances orientational order and is compatible with various positional orders of all the higher order phases (broken symmetries) up to and including the crystal phase. This is despite the globally random dispersion of CNTs in these nanocomposites and in contrast to the disordering effects observed in the $8 \mathrm{CB} / \mathrm{CNT}$ system [31]. Clearly, at such low concentrations with random CNT distribution, the specific CNT- LC boundary condition must play an important role along with the ultra-high aspect ratio (string-like structure) of the CNT nanoparticles.

To account for the opposing the behavior of the I-N transition between 9OO4/CNT and 8CB/ CNT given the similar chemical nature of both liquid crystals and common graphene surface, the CNT may promote nematic order in both liquid crystals but with different correlation lengths 
of the local anchoring field. For $8 \mathrm{CB} / \mathrm{CNT}$ system, the nucleated local director for $8 \mathrm{CB}$ varies in orientation along the long-axis of the CNT creating a surface paranematic layer while further away from the CNT surface, $\hat{n}$ becomes aligned parallel to the CNT strands [31]. This would mean that the local anchoring field direction varies along the CNT strands over a distance comparable to or shorter than the $\mathrm{n}$ correlation length, leading to quenched random-field disordering effects [33]. For 9OO4/CNT system, the surface anchoring orientation correlation length must be larger than the $\mathrm{n}$ correlation length of the LC, likely parallel to the $\mathrm{CNT}$ long axis with essentially no distortion (elastic strain) of $n$ further away from the strand surface. This would yield a quenched locally nonrandom field that spans more than one nematic domain, while still possessing a global (macroscopic) random distribution. This is consistent with the observed ECE in 9OO4/CNT and not in 8CB/CNT [46, 47], which would also account for the suppression of smectic order in the $8 \mathrm{CB} / \mathrm{CNT}$ system as the pinned surface para-nematic layer would further disorder any positional ordering. In 9OO4/CNT, the local field spans multiple nematic domains and would actually suppress director fluctuations and so promote smectic ordering.

However, the observed enhancement of the higher order smectic phases as well as the crystal phase implies that the uniform surface orientational anchoring is not accompanied by positional pinning along the CNT for the 9004 molecule. This degree of freedom of the LC molecule to essentially slide along the surface and each other parallel to the CNT long-axis is needed to accommodate all the higher positional ordering (broken symmetries) in pure 90O4, yielding the observed bulk-like behavior and phase boundary enhancement in this work. The origin of these two different behaviors between 9004 and $8 \mathrm{CB}$ with CNT is not immediately known but may be due to either the negative dielectric anisotropy of 9004 as opposed to that in $8 \mathrm{CB}$ or the different commensurate surface packing of the phenyl rings onto the graphene surface by these two LCs.

\section{Conclusions}

We have presented a detailed calorimetric study on the effect of carbon nanotubes on phase transitions of the 9OO4/CNT nanocomposites as a function of CNT concentration. The complex specific heat was measured over a wide range of temperature for negative dielectric anisotropy alkoxyphenylbenzoate liquid crystal (9OO4)/carbon nanotube (CNT) composites as a function of CNT concentration. The thermal scans were performed between 25 and $95^{\circ} \mathrm{C}$, first cooling followed by heating scans, as the function of CNT concentration ranging from 0 to $0.2 \mathrm{wt} \%$. All mesophases have transition temperatures $1 \mathrm{~K}$ higher and a crystallization temperature $4 \mathrm{~K}$ higher than that of the pure 90O4. The crystal phase superheats until a strongly first-order specific heat feature is observed, indicating melting $0.5 \mathrm{~K}$ higher than in the pure 9OO4. The enthalpy change associated with I-N and N-SmA phase transitions is only slightly changed with increasing $\varphi_{w}$ but are generally lower than pure 9OO4. The total transition enthalpy associated with the all transitions is independent on the CNT concentration and thermal treatment. The bulk or pure-like behavior of the phase transitions is supported by the bulk-like $9004 \mathrm{ECE}$ behavior of the SmA-SmC for the $0.05 \mathrm{wt} \%$ sample. We interpret that these results as arising from the LC-CNT surface interaction breaking orientational symmetry uniformly over a distance along the CNT greater than the nematic correlation length but allowing the LC to slide essentially freely on the CNT surface to accommodate various 
translational symmetries, leading to a net ordering effect for all transitions. These results suggest that the interactions between molecular structure, dipole moment of liquid crystal, and graphene-like surface can allow a random dispersion of CNT to promote both orientational and positional order depending on the length scale of the local order and the degree of surface freedom. The observed effects are incorporating CNTs with LC likely due to elastic coupling between CNT and LC and this leads to change in the elastic properties of composites. Continued experimental efforts probing the homogeneity of the sample, frequencydependent dynamics, smectic structures via X-ray scattering, and elastic behavior via light scattering of the homogeneous sample as a function of CNT concentration and temperature would be particularly important and interesting.

\section{Acknowledgements}

The authors are grateful to Robert P. Lemieux for providing the 9004 liquid crystal. This work was supported at WPI by the Department of Physics and a grant from the NSF award DMR0821292(MRI). At CWRU, this work was supported by the National Science Foundation's Condensed Matter Physics and Solid State and Materials Chemistry Programs under grant DMR-1065491.

\section{Author details}

Parvathalu Kalakonda* and Germano S Iannacchione

*Address all correspondence to: parvathalu.k@gmail.com

Department of Physics, Worcester Polytechnic Institute, Worcester, Massachusetts, USA

\section{References}

[1] Iijima S. Nature. 1991;354:56

[2] Thostenson ET, Ren ZF, Chou TW. Composites Science and Technology. 2001;61:1899

[3] Coleman JN, Khan U, Gun'ko YK. Advanced Material. 2006;18:689

[4] Coleman JN, Khan U, Blau WJ, Gun'ko YK. Carbon. 2006;44:1624

[5] Gojny FH, Wichmann MHG, Fiedler B, Kinloch IA, Bauhofer W, Windle AH, Schulte K. Polymer. 2006;47:2036

[6] Qunaies Z, Park C, Wise KE, Siochi EJ, Harrison JS. Composites Science and Technology. 2003;63:1637

[7] Haggenmueller R, Guthy C, Lukes JR, Fischer JE, Winey KI. Macromolecules. 2007;40:2417 
[8] Bhattacharyya AR, Sreekumar TV, Liu T, Kumar S, Ericson LM, Hauge RH, Smalley RE. Polymer. 2003;44:2373

[9] Assouline E, Lustiger A, Barber AH, Cooper CA, Klein E, Wachtel E, Wagner HD. Journal of Polymer Science Part B: Polymer Physics. 2003;41(520)

[10] Sandler JKW, Pegel S, Cadek M, Gojny FH, Es MV, Lohmar J, Blau WJ, Schulte K, Windle AH, Shaffer MSP. Polymer. 2004;45:2001

[11] Potschke P, Fornes TD, Paul DR. Polymer. 2002;43:3247

[12] Kharchenko SB, Douglas JF, Obrzut J, Grulke EA, Migler KB. Nature Material. 2004;3(564)

[13] Poncharal P, Wang ZL, Ugarate D, de Heer WA. Science. 1999;283:1513

[14] Wang L, Dang ZM. Applied Physics Letters. 2005;87:042903

[15] Xu Y, Zhang Y, Suhir E. Journal of Applied Physics. 2006;100:113524

[16] Zhao GL, Bagayoko D, Yang L. Journal of Applied Physics. 2006;99:114311

[17] Sinha S, Barjami S, Iannacchione G, Schwab A, Muench G. Journal of Nanoparticle Research. 2005;7:651

[18] Stephan O, Taverna D, Kociak M, Suenaga K, Henrard L, Colliex C. Physical Review B. 2002;66:155422

[19] Madarhri A, Pecastings G, Carmona F, Achour ME. Journal of Microwave Optoelectronics. 6, 3615.2007

[20] Sato Y, Terauchi M, Saito Y, Saito R. Journal of Electron Microscopy. 2006;55:137

[21] Potschke P, Dudkin SM, Alig I. Polymer. 2003;44:5023

[22] Ahmad K, Pan W, Shi SL. Polymer. 2006;55:133122

[23] Baughman RH, Zakhidov AA, de Heer WA. Science. 2002;297:787

[24] Collins PG, Avouris P. Scientific American. 2000;283:62

[25] Javey A, Wang Q, Urai A, Li Y, Dai H. Nano Letters. 2002;2:929

[26] Martel R, Schmidt T, Shea HR, Hertel T, Avouris P. Applied Physics Letters. 1998;73:2447

[27] Goodby JW, Waugh, Stein SM, Chin E, Pindak R, Patel JS. Nature. 1989;337:449

[28] Chandrashekhar S. Liquid Crystals. England: Cambridge University Press; 1992

[29] Demus D, Goodby JW, Gray G, Spiess H, Vill V, editors. Handbook of Liquid Crystals. Canada: Wiley-VCH; 1998

[30] de Gennes PG, Prost J. The Physics of Liquid Crystals. Clarendon, Oxford, England: Oxford University Press; 1993

[31] Sigdel KP, Iannacchione GS. European Physical Journal E. 2011;34:11034 
[32] Iannacchione GS, Park S, Garland CW, Birgeneau RJ, Leheny RL. Physical Review E. 2003;67:011709

[33] Iannacchione GS. Fluid Phase Equilibria. 2004;222/223:177

[34] Duran H, Gazdecki B, Yamashita A, Kyu T. Liquid Crystals. 2005;32:815

[35] Ji Y, Huang YY, Terentjev EM. Langmuir. 2011;27:13254

[36] Somoza AM, Sagui C, Roland C. Physical Review B. 2001;63, 081403(R)

[37] Song W, Windle AH. Macromolecules. 2005;38:6181

[38] Song W, Kinloch IA, Windle AH. Science. 2003;302:1363

[39] Zhang S, Kumar S. Small. 2008;4:1270

[40] Chen HY, Lee W, Clark NA. Applied Physics Letters. 2007;90:033510

[41] Chen HY, Lee W. Optical Review. 2005;12:223

[42] Basu R, Iannacchione GS. Physical Review E. 2010;81:051705

[43] Basu R, Boccuzzi KA, Ferjani S, Rosenblatt C. Applied Physics Letters. 2010;97:21908

[44] Basu R, Iannacchione G. Journal of Applied Physics. 2009;106:124312, 16

[45] Basu R, Chen CL, Rosenblatt C. Journal of Applied Physics. 2011;109:083518

[46] Basu R, Pendery JS, Petschek RG, Lemieux RP, Rosenblatt C. Physical Review Letters. 2011;104:237804

[47] Basu R, Rosenblatt C, Lemieux RP. Liquid Crystals. 2012;39:199

[48] P. Kalakonda and G. S. Iannacchione, to be submitted (2013)

[49] Schawe JEK. Thermochimica Acta. 1995;260:1

[50] Coleman NJ, Craig DQM. International Journal of Pharmaceuticals. 1996;135:13

[51] Hutchinson JM, Montserrat S. Journal of Thermal Analysis. 1996;47:103

[52] Hensel A, Dobbertin J, Boller A, Schawe JEK. Journal of Thermal Analysis. 1996;46:935

[53] Wunderlich B. Journal of Thermal Analysis. 1997;48:207

[54] Aubuchon SR, Gill PS. Journal of Thermal Analysis. 1997;49:1039

[55] Weyer S, Hensel A, Schick C. Thermochimica Acta. 1997;304/305:267

[56] Schawe JEK. Thermochimica Acta. 1996;271:127

[57] Garoff S, Meyer RB. Physical Review Letters. 1977;38:848

[58] Berardi R, Kuball HG, Memmer R, Zannon C. Journal of the Chemical Society, Faraday Transactions. 1998;94:1229

[59] Molecular modeling was done using Avogadro: An open-source molecular builder and visualization tool. Version 1.XX. http://avogadro.openmolecules.net/. 17 